\title{
DIE MUSIK
}

DES

\section{GRIECHISCHEN ALTERT'HUMES.}

NACH DEN ALTEN QUELLEN NEU BEARBEITET

VON

RUDOLF WESTPHAL.

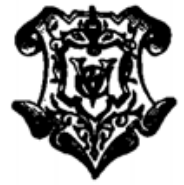

LEIPZIG,

VERLAG VON VEIT \& COMP.

1883. 
Das Recht der Herausgabe von Uebersetzungen vorbehalten.

Druck von Metzger \& Wittig in Leipzig. 
DE N H E R E N

\section{H. WEIL,}

MITGIIED VER ACADF́MIE DES INSCRIPTIONS ET BELLBS-LETTRES ZU PARIS,

\section{CH. ÉM. RUELLE,}

RIJLIOTHEKAR VON ST. GENOFEVA ZU PARIS,

\section{F. A. GEVAERT,}

VORNKM DIRKCTOR DER GROSSISN OHER ZU PARIS,

GEGENWÄRTIG DIRECTOR DES CONSERVATORIUMS ZU BRÜSSEL,

\section{SEI DIESES BUCH}

DANKBARLICHST GEWIDMET. 
